\title{
BMJ Open Youth participatory research evidence to inform health policy: a systematic review protocol
}

\author{
Janet Njelesani (D) ,1,2 Jean Hunleth ${ }^{3}$
}

To cite: Njelesani J, Hunleth J. Youth participatory research evidence to inform health policy: a systematic review protocol. BMJ Open 2020;10:e036522. doi:10.1136/ bmjopen-2019-036522

\section{- Prepublication history and} additional material for this paper are available online. To view these files, please visit the journal online (http://dx.doi org/10.1136/bmjopen-2019036522).

Received 18 December 2019 Revised 04 May 2020 Accepted 01 July 2020

\section{Check for updates}

(C) Author(s) (or their employer(s)) 2020. Re-use permitted under CC BY-NC. No commercial re-use. See rights and permissions. Published by BMJ.

${ }^{1}$ Occupational Therapy, New York University, New York, New York, USA

${ }^{2}$ International Centre for Disability and Rehabilitation, University of Toronto, Toronto, Canada

${ }^{3}$ Division of Public Health Sciences, Washington University in Saint Louis, Saint Louis, Missouri, USA

Correspondence to Dr Jean Hunleth; jean.hunleth@wustl.edu

\section{ABSTRACT}

Introduction Young people's participation in health research produces knowledge that is indispensable for creating appropriate and effective policies. However, how best to disseminate youth participatory research evidence to impact health policy is not known. Therefore, the objectives of this systematic review are to describe the evidence produced through youth participatory research, including the strategies used to disseminate youth participatory research evidence to health policymakers. These are necessary to improve policymakers' use of youth participatory research evidence and, thereby, make programmes more impactful for young people.

Methods and analysis The meta-narrative methodology will guide the systematic review to highlight the contrasting and complementary evidence on the use of engaging youth in research to affect health policymaking. Relevant studies will be identified by searching electronic databases, including but not limited to EBSCO, PROQUEST, OVID Medline, Sociological Abstracts and Google Scholar from inception to December 2020. The methodological quality of included quantitative, qualitative and mixedmethods research studies will be assessed using valid appraisal tools. The meta-narrative approach to analysis will include identifying meta-narratives of how youth participation informed the health research findings. Ethics and dissemination An advisory group of young people will advise on the study and dissemination of the findings. As part of our plan for active dissemination, we will produce a policy brief that builds the rationale for using research with and by youth as part of an evidence base necessary for achieving youth health outcomes.

\section{INTRODUCTION}

Young people's participation in research produces knowledge that is indispensable for creating appropriate and effective policies and practices. ${ }^{1-3}$ The absence of young people's input can lead to ineffective and potentially detrimental policies. ${ }^{45}$ Young people can productively engage in the planning, design and implementation of research. ${ }^{6} 7$ Their participation in research provides a form of evidence unavailable through other research means (eg, interviewing adults, document analysis) ${ }^{8}$ as adults (eg, parents) are not good proxies for young people's perceptions

\section{Strengths and limitations of this study}

This study will be the first to systematically assess the literature on youth participatory research evidence for informing health policy.

- Involving a Youth Advisory Group throughout the review, will ensure youth participation and strengthen the relevance of the results.

- The review will be limited to studies written in English, which may result in exclusions of studies published in other languages.

and actions. While researchers use a range of techniques to increase youth participation, participatory research, ${ }^{9}$ especially as part of an ethnographic approach, ${ }^{10-14}$ and youth-led participatory action research (YPAR) ${ }^{15}$ are leading research approaches with young people. In YPAR, young people investigate, document and analyse issues, and develop and implement action plans to rectify those issues. ${ }^{16}$ Participatory research and YPAR acknowledge and attempt to address power imbalances that privilege researcher and adult perspectives and agendas and challenge top-down policy development, privileging instead 'inside knowledge'. ${ }^{17}$

In health research, the participation of adult stakeholders has increased. ${ }^{18}$ Adult participants are co-designing research and co-constructing knowledge. As a result, participatory research is informing health policy through research centres and institutes, such as the Patient Centered Outcomes Research Institute. Previous systematic reviews on the impact of adult-stakeholder engagement in research concluded that engagement is feasible in health settings and enhances the quality and appropriateness of research and policy. ${ }^{19}$ Previous systematic reviews have also focused on youth engagement in health research. $^{2021}$ This literature has primarily focused on ethical issues and risks associated with participation ${ }^{22}$ and on developing methods to include young people as research 
participants. ${ }^{23}$ However, to our knowledge, no systematic review has synthesised evidence on the implementation of youth-derived health research evidence to inform health policy. Such a review is a critical step towards shifting youth health policy to become more sensitive to youth's needs and experiences. Therefore, the objectives of this systematic review are to describe the evidence produced through youth participatory research for informing policy, as well as the strategies used to disseminate youth participatory research evidence to health policymakers. These are necessary first steps to improve policymakers use of youth participatory research evidence and, thereby, make programmes more impactful for young people.

This systematic review focuses on youth participatory research evidence in health. The field of health was chosen because it is a broad field that will allow for a comprehensive, interdisciplinary exploration and a growing number of health researchers are using youth participatory research as a methodological approach. ${ }^{24}$ Furthermore, our recent scoping review of youth inclusion in health intervention research showed an increase over the past decade. We also identified gaps in dissemination of youth-produced evidence and a tokenisation of youth participatory research evidence. ${ }^{25}$ These findings demonstrate a need for better awareness of youth participatory research evidence and strategies to disseminate it.

\section{Purpose}

We will carry out a systematic review to describe the evidence produced through youth participatory research and its use for informing health policy, and to determine systematic and replicable strategies that researchers use to disseminate youth participatory research evidence to health policymakers.

\section{METHODS AND ANALYSIS}

Health research spans different fields and disciplines and uses diverse terms and definitions. Therefore, we will use the meta-narrative methodology developed by Greenhalgh $^{26}$ as a systematic, theory-driven, interpretative approach to make sense of heterogeneous evidence applied in diverse contexts. The approach is highly suited to our review, as it will highlight the contrasting and complementary evidence on the use of engaging youth in research to affect health policymaking. Additionally, this systematic review protocol follows Preferred Reporting Items for Systematic Review and Meta-Analysis Protocols (PRISMA-P) $2015^{27}$ reporting guideline (see online supplementary file 2 for PRISMA-P 2015 Checklist) as well as the data extraction processes outlined by the Cochrane Collaboration. ${ }^{28}$

\section{Stage 1: planning phase}

All planning will follow the meta-narrative methodology protocol and will be designed to achieve the objectives. In an in-person planning meeting, we will share plans with and seek guidance from our Youth Advisory Group (ages 15-25). In this phase, youth will be involved in confirming the aim and priorities of the review. This active group brings expertise on health through their personal and professional experiences (eg, members of Disabled Persons Organizations), and has a good rapport with the team having worked together on previous health studies.

\section{Stage 2: search and mapping phase}

Published studies that report the utilisation of youth participatory research to inform health policy will be included. Inclusion criteria will encompass studies in English of any design, level of evidence, or paradigm and systematic reviews. We will limit our search to young people (ages 5-25) and exclude grey literature. There will be no time frame restrictions, so databases will be searched from inception to December 2020. We will exclude all studies where young people's involvement is deemed as non-participation based on Hart's ${ }^{29}$ Ladder of Youth Participation. Non-participation (Hart's rungs 1-3) includes tokenism, participation as decoration and participation that adults manipulate. Hart's depiction of active participation ranges widely from youth assigned a role in a researcher-led project (rung 4) to youth initiating projects and sharing decision-making (rung 8). A YPAR focus fits more fully with the higher rungs in Hart's Ladder (eg, 6, where researchers share decisions about the study with youth). However, our previous experience with using Hart's Ladder to classify studies has taught us that we must include the lower rungs of participation (eg, 4 and 5) so as not to exclude important research questions and participants (eg, youth who by desire, ability or circumstance cannot participate in decision-making). If participation versus non-participation cannot be discerned based on the criteria in the article, we will contact the corresponding author to invite them to submit more information on study methodology.

We will refine our search strategy in conjunction with an expert librarian familiar with youth participatory work. Relevant studies will be identified by searching electronic databases including but not limited to EBSCO, PROQUEST, OVID Medline, Sociological Abstracts and Google Scholar. Keywords will be identified in conjunction with the expert librarian based on standard indexing practices and include health AND policy AND youth or child or young person AND participatory research or action research or engagement (see online supplementary file 1 for search strategy of OVID Medline). To identify additional studies, reference lists from eligible studies will be searched and key journals will be hand searched. We will export identified records to Covidence, as recommended by Cochrane, ${ }^{28}$ so the review team can collaborate from anywhere. Study titles and abstracts will first be screened to determine if they meet inclusion criteria. Full-texts of potentially relevant papers will then be evaluated and reasons for exclusion recorded. Paired reviewers will screen title, abstract and full-text independently. A third reviewer will resolve disagreements as needed. 


\section{Stage 3: appraisal phase}

Depending on study methods, we will appraise study quality using the Quality Assessment Tool for Observational Cohort and Cross-Sectional Studies for quantitative studies, ${ }^{30}$ the Critical Appraisal Skills Programme Qualitative Checklist for qualitative studies ${ }^{31}$ and the Mixed Methods Appraisal Tool for mixed-method studies. ${ }^{32}$ These specific tools were chosen based on their fit with the study designs included in our review, validity, evidence for use, practicality and our previous experience using them.

A data abstraction sheet will be developed using a customised Covidence form and include the general details of each study and details specific to the review objectives: that is, phenomena of interest; methodology; descriptions of young people, including social, physical and developmental factors, age, gender, ethnicity, dis/ ability; youth participation methods; level of youth participation $^{29}$; context; youth outcomes; policy relevance to youth; identified dissemination strategies (eg, passive, active). We will also extract the degree of end-user (ie, policymaker) involvement in the research as this is known to increase the use of research evidence. ${ }^{33}$ To enhance rigour, two independent extractors will use the Covidence form and compare completed abstraction forms.

\section{Stage 4: data synthesis phase}

Due to the study objectives and heterogeneity of sources, a meta-narrative approach to analysis will be conducted using the software package NVivo V.12, and data will be systematically thematically analysed. ${ }^{26}$ First, we will identify meta-narratives by reading the extracted data and coding (ie, reoccurring ideas that are conceptually related will be grouped into concepts) to describe how the evidence produced from young people's participation in research was used to inform policy. Iterative rounds of inductive and deductive coding will be used. Identified meta-narratives will then be systematically applied to all papers. On drafting preliminary results, a participatory workshop will be held with the Advisory Group to invite their critical reflection on the results and determine their priorities for the dissemination activities.

Alongside providing extracted details (Stage 3), we will develop inductive codes of how youth participation informed the findings. These inductive codes will offer an evidence base to build a rationale for youth participatory research evidence (step 1 in the framework for the dissemination of evidence). ${ }^{34}$ Such an evidence base and gaps that need further research are only established through reviews. 35

To identify strategies that researchers use to disseminate youth participatory research evidence to health policymakers, we will use the deductive code, 'dissemination strategies,' and code in NVivo12 all content related to dissemination to policymakers. We will assess this code further and compile a list of frequency of strategies and stated facilitators and barriers to dissemination. As per the Model for Dissemination of Research, ${ }^{36}$ we will account for the message, source (eg, researchers, youth participants, policymakers), audience (ie, type of policymaker) and channel (eg, meetings, workshops, news, social media) in each dissemination strategy.

\section{ETHICS AND DISSEMINATION}

Ethical approval is not required, as primary data will not be collected. We plan to conduct a comprehensive and reproducible review of the literature while recognising that there may be some limitations. Including, language limitations of the research team prevent including studies published in languages other than English. Further, some studies may be missed in our search strategy, though we have an experienced librarian on the team to ensure that our search strategy is as inclusive as possible.

We anticipate that youth participatory research evidence does not yet affect policy, in part, because of a lack of a central framework for disseminating findings, the lack of youth involvement in driving dissemination, attitudes towards youth and a reliance on ineffective passive dissemination techniques (eg, presenting at academic conferences, publishing in academic journals). Dissemination and Implementation Science provides frameworks for understanding how research evidence can best be communicated to improve uptake and use. Specifically relevant are dissemination frameworks based on Diffusion of Innovations theory, ${ }^{37}$ which is widely used to explain the processes that influence the use of research evidence $^{38}$ and also understandings from anthropology that information does not flow unidirectionally and that people (eg, policymakers, in this case) creatively reinvent and reinterpret such evidence, which contributes to change. By synthesising the health research, our review will build rationale and credibility for greater use of youth participatory research evidence as well as a better understanding of the dissemination of evidence produced by young people.

The systematic review will create a path forward for the dissemination of youth participatory research evidence to health policymakers, particularly those at state and local levels. Therefore, active dissemination is critical. ${ }^{34}$ In particular, we will develop policy briefs to build a brief that builds the rationale for using research with and by youth as part of an evidence base necessary for achieving youth health outcomes.

\section{PATIENT AND PUBLIC INVOLVEMENT}

Public involvement is central to the work, as a Youth Advisory Group will be involved throughout the process as described in the "Methods and analysis" section.

Contributors JN and $\mathrm{JH}$ were responsible for the conceptualisation and development of all components of the review. Together they co-developed, then revised the first draft of the manuscript. Both authors read and approved the final manuscript.

Funding $\mathrm{JH}$ received funding from the Foundation for Barnes Jewish Hospital and the Siteman Cancer Center for time spent conceptualising this research and the writing of this article. 
Competing interests None declared.

Patient consent for publication Not required.

Provenance and peer review Not commissioned; externally peer reviewed.

Open access This is an open access article distributed in accordance with the Creative Commons Attribution Non Commercial (CC BY-NC 4.0) license, which permits others to distribute, remix, adapt, build upon this work non-commercially, and license their derivative works on different terms, provided the original work is properly cited, appropriate credit is given, any changes made indicated, and the use is non-commercial. See: http://creativecommons.org/licenses/by-nc/4.0/.

ORCID iD

Janet Njelesani http://orcid.org/0000-0002-6134-3950

\section{REFERENCES}

1 Torre ME, Fine M. Participatory action research (PAR) by youth In: Youth activism: an international encyclopedia. Westport, CT: Greenwood, 2006: 456-62.

2 Quijada Cerecer DA, Cahill C, Bradley M. Toward a critical youth policy praxis: critical youth studies and participatory action research. Theory Pract 2013;52:216-23.

3 Cammarota J, Romero A. Participatory action research for high school students: transforming policy, practice, and the personal with social justice education. Educ Policy 2011;25:488-506.

4 Hunleth J. "ARVs" as sickness and medicine: examining children's knowledge and experience in the HIV era in urban Zambia. AIDS Care 2013;25:763-6.

5 Spray J. The value of anthropology in child health policy. Anthropology in Action 2018;25:29-40.

6 Hunleth J. Children's roles in tuberculosis treatment regimes: constructing childhood and kinship in urban Zambia. Med Anthropol Q 2013b;27:292-311.

7 Njelesani J. "A child who is hidden has no rights": responses to violence against children with disabilities. Child Abuse Negl 2019;89:58-69.

8 Njelesani J, Leckie K, Drummond J, et al. Parental perceptions of barriers to physical activity in children with developmental disabilities living in Trinidad and Tobago. Disabil Rehabil 2015;37:290-5.

9 Aldridge J. Participatory research: working with vulnerable groups in research and practice. Chicago, IL: Bristol University Press, 2015.

10 Hunleth J. Beyond on or with: questioning power dynamics and knowledge production in 'child-oriented' research methodology. Childhood 2011;18:81-93.

11 Hunleth J. Children as caregivers: the global fight against tuberculosis and HIV in Zambia. New Brunswick, NJ: Rutgers University Press, 2017.

12 Hunleth J. Zambian children's imaginal caring: on fantasy, play, and anticipation in an epidemic. Cultural Anthropology 2019;34:155-86.

13 Njelesani J, Sedgwick A, Davis JA, et al. The influence of context: naturalistic study of Ugandan children's doings in outdoor spaces. Occup Ther Int 2011;18:124-32.

14 Njelesani J, Hashemi G, Cameron C, et al. From the day they are born: a qualitative study exploring violence against children with disabilities in West Africa. BMC Public Health 2018;18:153.

15 Ergler CR. Advocating for a more relational and dynamic model of participation for child researchers. Social Inclusion 2017;5:240-50.

16 Cammarota J, Fine M. Youth participatory action research: a pedagogy for transformational resistance. In: Revolutionizing education: youth participatory action research in motion. Routledge Taylor \& Francis Group, 2008: 1-11.

17 Silver LJ. Transformative childhood studies - a remix in inquiry, justice, and love. Children's Geographies2019:1-15.

18 Brett J, Staniszewska S, Mockford C, et al. A systematic review of the impact of patient and public involvement on service users researchers and communities. Patient 2014;7:387-95.
19 Oliver S, Harden A, Rees R, et al. An emerging framework for including different types of evidence in systematic reviews for public policy. Evaluation 2005;11:428-46.

20 Vaughn LM, Wagner E, Jacquez F. A review of community-based participatory research in child health. MCN Am J Matern Child Nurs 2013;38:48-53.

21 Anyon $\mathrm{Y}$, Bender $\mathrm{K}$, Kennedy $\mathrm{H}$, et al. A systematic review of youth participatory action research (YPAR) in the United States: methodologies, youth outcomes, and future directions. Health Educ Behav 2018;45:865-78.

22 Huang X, O'Connor M, Ke L-S, et al. Ethical and methodological issues in qualitative health research involving children: a systematic review. Nurs Ethics 2016;23:339-56.

23 Kirk S. Methodological and ethical issues in conducting qualitative research with children and young people: a literature review. Int J Nurs Stud 2007;44:1250-60.

24 Boote J, Wong R, Booth A. 'Talking the talk or walking the walk?' a bibliometric review of the literature on public involvement in health research published between 1995 and 2009. Health Expect 2015;18:44-57.

25 Hunleth J, Spray J, Meehan C. Children's meaningful participation in implementation research on health interventions: a scoping study, 2020.

26 Greenhalgh T, Robert G, Macfarlane F, et al. Storylines of research in diffusion of innovation: a meta-narrative approach to systematic review. Soc Sci Med 2005;61:417-30.

27 Moher D, Shamseer L, Clarke M, et al. Preferred reporting items for systematic review and meta-analysis protocols (PRISMA-P) 2015 statement. Syst Rev 2015;4:1.

28 Cochrane Community. Covidence, 2019. Available: http://community. cochrane.org/tools/review-production-tools/covidence.

29 Hart R. Children's participation: from tokenism to citizenship, Innocenti essay No. 4. Florence: International Child Development Centre, 1992

30 National Institutes of Health. Quality assessment tool for observational cohort and cross-sectional studies, 2019. Available: https://www.nhlbi.nih.gov/health-pro/guidelines/in-develop/ cardiovascular-risk-reduction/tools/cohort

31 Critical Appraisal Skills Programme. 10 questions to help you make sense of qualitative research, 2013. Available: http://media.wix.com/ ugd/dded87_29c5b002d99342f788c6ac670e49f274.pdf

32 Pluye P, Hong QN, Vedel I. Toolkit for mixed studies reviews (V3). Montreal, Canada: Department of Family Medicine, McGill University, and Quebec-SPOR SUPPORT Unit, 2016. http://toolkit4mixedstu diesreviews.pbworks.com

33 Jagosh J, Pluye P, Macaulay AC, et al. Assessing the outcomes of participatory research: protocol for identifying, selecting, appraising and synthesizing the literature for realist review. Implement Sci 2011;6:24.

34 Purtle J, Dodson EA, Brownson RC. Policy dissemination research. In: Brownson RC, Colditz G, Proctor EK, eds. Dissemination and implementation research in health. 2nd edn. New York: Oxford University Press, 2018.

35 Oliver K, Innvar S, Lorenc T, et al. A systematic review of barriers to and facilitators of the use of evidence by policymakers. BMC Health Serv Res 2014;14:2

36 Brownson RC, Colditz GA, Proctor EK. Dissemination and implementation research in health: translating science to practice. 2nd edn. New York: Oxford University Press, 2018.

37 Rogers EM. Diffusion of innovation. 5th edn. New York, NY: Free Press, 2003.

38 Rabin BA, Brownson RC. Developing the terminology for dissemination and implementation research. In: Brownson RC, Colditz G, Proctor EK, eds. Dissemination and implementation research in health. 2nd edn. New York: Oxford University Press, 2018. 